\title{
Aproximación al coste de políticas públicas de apoyo a la financiación del emprendimiento en cooperativas españolas ${ }^{1}$
}

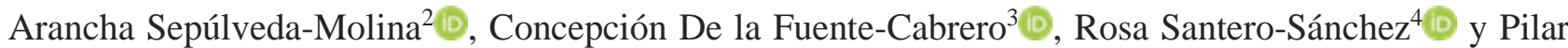 \\ Laguna-Sánchez ${ }^{5}$ (†)
}

Recibido: 5 de noviembre de 2021 / Aceptado: 11 de enero de 2022 / Publicado: 14 de febrero de 2022

Resumen. La contribución de las cooperativas al cumplimiento de los Objetivos de Desarrollo Sostenible ha sido reconocida a nivel internacional. La Agenda 2030 recoge la necesidad de implementar políticas públicas amplias y efectivas que fomenten el desarrollo de las entidades de Economía Social para maximizar su impacto. Ahora bien, para poner en marcha nuevos proyectos cooperativos de actividades ligadas al desarrollo sostenible va a ser necesario movilizar también créditos bancarios, una de las fuentes más relevantes de financiación para los emprendedores españoles; y su obtención suele estar sujeta a la aportación de garantías, lo cual puede representar un problema. Esta dificultad se puede mitigar con las Sociedades de Garantía Recíproca (SGR). El objetivo de esta investigación es analizar la ratio de fallido de préstamos avalados a cooperativas de nueva creación por las SGR españolas entre los años 2003 y 2012 . El periodo es relevante porque abarca un periodo de expansión hasta el año 2008 y crisis económica posterior a este año. Los resultados revelan un mejor comportamiento de las cooperativas en relación con otras formas empresariales y permiten comparar, por diferentes criterios de clasificación (tamaño de la empresa, sector de actividad y destino de los fondos), el coste del fallido de la cartera global y del correspondiente a las cooperativas, observándose en general una situación más favorable para estas últimas, con ratios inferiores. Las conclusiones son relevantes para los gestores de SGR, las entidades financieras, y especialmente para las Administraciones Públicas y se pueden tener en cuenta para estimar la aportación de fondos de provisiones técnicas destinados a apoyar la financiación de iniciativas emprendedoras sostenibles a través de cooperativas.

Palabras clave: Financiación de cooperativas; Sociedades de Garantía Recíproca; Préstamos garantizados; Coste del fallido; Emprendimiento.

Claves Econlit: G01; G210; L26; J54.

\section{[en] Approach to the cost of public policies to support the financing of entrepreneurship in cooperatives}

\begin{abstract}
The contribution of cooperatives to the attainment of the Sustainable Development Goals is recognised at an international level. The 2030 Agenda includes the need to implement comprehensive, effective public policies that promote the development of Social Economy organisations to maximise their impact. One of the most important sources of financing for Spanish entrepreneurs is bank loans, then these credits are going to be necessary for launching new cooperative projects linked to sustainable development activities. However, obtaining them is usually subject to the provision of guarantees, which can be a setback. This difficulty can be mitigated by Mutual Guarantee Institutions (MGI). The aim of this research is to analyse the failure ratio of loans guaranteed to newly created cooperatives by Spanish MGIs between 2003 and 2012. This period is pertinent as it covers a period of expansion until 2008 followed by the economic crisis. The results revealed that cooperatives performed better than other business models and permit a comparison, considering various classification criteria (size of the company, sector of activity and destination of funds), of the cost of default of the overall portfolio and that corresponding to cooperatives, with a generally more favourable situation being observed for the latter, as they present lower ratios. The conclusions are relevant for MGS managers, financial institutions and especially for public administrations, and can be considered when estimating the contribution of technical provision funds to support the financing of sustainable entrepreneurial initiatives through cooperatives.
\end{abstract}

Keywords: Cooperatives financing; Mutual Guarantee Institutions; Guaranteed loans; Default cost; Entrepreneurship.

1 Agradecimientos: A la Compañía Española de Reafianzamiento SA (CERSA) por suministrar la base de datos que ha hecho posible esta investigación.

2 Universidad Rey Juan Carlos, España

Dirección de correo electrónico: a.sepulvedam@alumnos.urjc.es.

Universidad Rey Juan Carlos, España.

Dirección de correo electrónico: concepcion.delafuente@urjc.es

4 Universidad Rey Juan Carlos, España.

Dirección de correo electrónico: rosa.santero@urjc.es

5 Universidad Rey Juan Carlos, España.

Dirección de correo electrónico: pilar.laguna@urjc.es 
Sumario. 1. Introducción. 2. Metodología. 3. Resultados. 4. Conclusiones. 5. Referencias bibliográficas.

Cómo citar. Sepúlveda-Molina, A.; De la Fuente-Cabrero, C.; Santero-Sánchez, R.; Laguna-Sánchez, P. (2022) Aproximación al coste de políticas públicas de apoyo a la financiación del emprendimiento en cooperativas españolas. REVESCO. Revista de Estudios Cooperativos, vol. 140, e79942. https://dx.doi.org/10.5209/reve.79942.

\section{Introducción}

Las cooperativas, al igual que otras formas empresariales de la Economía Social, son organizaciones que desarrollan su actividad impulsadas por valores y principios, como la equidad, la solidaridad, la democracia, el compromiso con el medio ambiente, que ponen en el centro a las personas, por encima del capital, y, para las que el desarrollo sostenible forma parte de su naturaleza (Fernández-Guadaño, López-Millán y SarriaPedroza, 2020).

Los principios cooperativos están alineados con los Objetivos de Desarrollo Sostenible (ODS) de la Agenda 2030 de la ONU (Naciones Unidas, 2015). La contribución de las cooperativas al cumplimiento de los ODS ha sido reconocida a nivel político por organismos internacionales, como el Grupo de Trabajo de las Naciones Unidas sobre Economía Social y Solidaria (United Nations Task Force on Social and Solidarity Economy) y la Alianza Internacional Cooperativa, y en el ámbito de la investigación, la evidencia científica muestra una alineación de la actuación de las cooperativas con la consecución de los ODS (Bastida, Vaquero García, Cancelo Márquez y Olveira Blanco, 2020; Castro-Nuñez, Bandeira y Santero-Sánchez, 2020; Mozas Moral y Puente Poyato, 2010, entre otras).

Por otra parte, las empresas de Economía Social, y en concreto las cooperativas, surgen cada vez más como una de las alternativas más viables al desarrollo sostenible en todos sus ámbitos (económico, social y medioambiental).

En relación con la sostenibilidad económica, algunos estudios realizados a nivel nacional (Atienza-Montero y Rodríguez-Pacheco, 2018), y autonómico, en comunidades como el País Vasco o Comunidad Valenciana (Zubiaurre, Andicoechea y Saitua, 2016; Pozuelo- Campillo, Carmona- Ibañez y Martínez- Vargas, 2012) muestran que las cooperativas cuentan con mejores ratios económico-financieros respecto a las PYMEs convencionales, además de tener mayores niveles de competitividad y productividad, debido principalmente a dos factores internos: la participación de los socios trabajadores en el proceso productivo y de comercialización de los productos, y la potenciación de la relación que mantienen las cooperativas con sus clientes (Moyano Fuentes, Puig Blanco, Bruque Cámara, 2008).

Desde un punto de vista social, la contribución de las cooperativas se produce en diferentes niveles. Podríamos hablar, por ejemplo, de su contribución al desarrollo económico y social de las zonas en las que llevan a cabo su actividad. Observamos que entre los principios básicos en los que está basado el cooperativismo, se encuentra el del interés por su comunidad. Este principio lleva implícito el hecho de contribuir al desarrollo de las zonas en las que las cooperativas operan, promoviendo un modelo de empresa sostenible, anclado en su entorno y fundamentado en el trabajo común para lograr un beneficio colectivo (Díaz Duque, 2013). Así mismo, contribuyen al desarrollo del denominado capital social, donde las cooperativas se interrelacionan con otras cooperativas y empresas, con clientes y proveedores dentro del entorno donde desarrollan su actividad (Bretos, Díaz Foncea, Marcuello y Marcuello, 2018).

En términos de empleo, estas empresas favorecen la inserción sociolaboral de grupos con riesgo de exclusión laboral y social, como inmigrantes, mujeres, personas con discapacidad, que suelen tener dificultades en el acceso al mercado de trabajo (Calderón Milán, Calderón Milán y Barba Sánchez, 2020; Castro-Nuñez et al., 2020; Melián Navarro y Campos Climent, 2010). Además, el empleo creado por las sociedades cooperativas es de mayor calidad y más estable en épocas de recesión, dado que depende menos del PIB del país en el que se encuentran instaladas, a diferencia del empleo creado por el resto de las compañías (Díaz Foncea, y Marcuello Servós, 2010).

Por último, cabe señalar que existen pocos estudios que analicen el impacto de las cooperativas sobre el medio ambiente. La investigación empírica en esta línea se ha centrado en cooperativas agrícolas y agroalimentarias principalmente y en el análisis de medidas que fomenten la sostenibilidad ambiental, como mejoras en la gestión del agua, la elección de cultivos más resistentes que eviten los efectos del calentamiento del planeta y el fomento del ahorro energético.

Actualmente, España, junto con el resto de los países de la Unión Europea, está inmersa en un proceso de recuperación económica, en el que se está movilizando una gran cantidad de recursos financieros (programa Next GenerationEU) que, con distintos instrumentos, pretende conseguir una Europa más ecológica, más digital, más resiliente y mejor adaptada a los retos actuales y futuros. En este entorno, las PYMEs españolas deberán recurrir a distintas fuentes de financiación, siendo la bancaria la más habitual. Actualmente existe una alta dependencia de las PYMEs con respecto a este tipo de financiación, siendo los préstamos bancarios uno de los instrumentos financieros más utilizados por los emprendedores españoles (Hernández, 2014; CESGAR, 
2019), con la particularidad de que su obtención suele estar sujeta a la aportación de avales o garantías. Para solventar este problema surgen los sistemas de garantía, que a veces se articulan en torno a las Mutual Guarantee Institutions (MGI), como se denomina en algunos países europeos, o las SGR españolas, que con su garantía apoyan la financiación de nuevos proyectos empresariales (Santero-Sánchez, De la Fuente-Cabrero, Laguna-Sánchez, 2016, Pombo, Molina y Ramírez, 2006, 2007). El aval de las SGR no solo posibilita el acceso al crédito, sino que mejora sus condiciones en cuanto a plazo y a tipo de interés, de los préstamos bancarios a las PYME (De la Fuente-Cabrero, 2007; Pombo, Molina y Ramírez, 2008; Columba, Gambacorta y Mistrulli, 2010; Bartoli, Ferri, Murro y Rotondi, 2013; De la Fuente-Cabrero, Laguna-Sánchez y Villacé-Molinero, 2013). Además, las SGR en España han tenido un importante papel durante la crisis de la COVID-19, facilitando el acceso a financiación adicional, proporcionando mejores condiciones de la financiación empresarial, prestándoles apoyo en la restructuración del pasivo, y evitando el cierre de empresas (CESGAR, 2021).

El movimiento cooperativista ha experimentado un continuo crecimiento desde sus inicios debido a sus políticas integradoras, democráticas y sociales que convierten a las cooperativas en un medio de subsistencia, especialmente en épocas de crisis (Belmonte, Guerrero, y Cabrera, 2018). En España, el sector cooperativo ha seguido una evolución positiva, con un crecimiento exponencial en su peso sobre el PIB y en el empleo generado, extendiéndose además a otros sectores más allá del agrícola (Díaz Foncea y Marcuello, 2013) y con una importante expansión internacional (Bretos, Díaz-Foncea y Marcuello, 2020).

La literatura científica sobre financiación de cooperativas se ha centrado fundamentalmente en los recursos propios como el propio capital social, excedentes no repartidos, las participaciones especiales, cuotas de ingreso y periódicas, títulos participativos o cuentas en participación (Gadea, 2005; Sánchez Pachón, 2016); y otras fuentes de financiación externa, como las subvenciones o la financiación bancaria (López Cózar y Priede, 2015). Hasta el momento, no se han encontrado estudios que se ocupen en profundidad de la financiación de sociedades cooperativas a través de las SGR, por lo que el trabajo que aquí se presenta, cubre una laguna dentro del análisis del sector cooperativo en España.

Las investigaciones sobre los sistemas de garantía, tanto a nivel nacional como internacional, analizan el impacto de estas instituciones desde distintas perspectivas. En primer lugar, destacan los trabajos que analizan las características de los diferentes sistemas de garantía, así como el papel que desempeñan como solución a las barreras de acceso al crédito de las PYME (Pombo et al., 2006, 2007, 2008; Fuente-Cabrero, 2007; Columba et al., 2010, Cowling, 2010; Escribano Sotos y Pardo García, 2011; Bartoli et al. 2013; Povedano, Sobrino, González, y Sánchez, 2013). En un segundo grupo, se encuentran aquellos trabajos que evalúan el papel que desempeña la garantía para mitigar los problemas de información asimétrica a los que se enfrentan los bancos (Parker, 2002; Taghizadeh Hesary, Yoshino, 2019). Otro grupo relevante de literatura se centra en las mejoras de las condiciones de los créditos, tanto en costes como en plazo (Wang, Zhang, Liu, Cullinan, 2016), así como en la disminución de los requerimientos realizados por parte de las entidades de crédito a las empresas (Briozzo, Cardone Riportella, Trujillo Ponce, 2013).

Otra parte de esta literatura refleja el impacto que tiene la obtención de préstamos garantizados desde el punto de vista de la empresa, en términos de crecimiento y desarrollo de la misma (Bertoni, Martí y Reverte, 2019), en su supervivencia en épocas de crisis (Bartoli, Ferri, Murro, Rotondi, 2013) y en la consecución de ventajas competitivas (Pergelova y Angulo-Ruiz, 2014).

Un estudio reciente para España estima el coste del fallido para la cartera de operaciones de financiación avaladas a pequeñas empresas de nueva creación por parte de las SGR entre los años 2003 y 2012 (De la Fuente-Cabrero, Castro-Pardo, Santero-Sánchez y Laguna-Sánchez, 2019b). Este trabajo aporta información relevante para los gestores de SGR y para las administraciones públicas, sobre posibles costes en las políticas de apoyo a la financiación de nuevos proyectos empresariales. Avanzando en esta misma línea, se desarrolla esta investigación, cuyo objetivo principal es analizar el ratio de fallido de una cartera de préstamos avalados por SGR españolas a cooperativas de nueva creación entre los años 2003 y 2012. Cabe destacar que las conclusiones de este estudio, son además relevantes para trabajar en la línea que pide la Agenda 2030 de Naciones Unidas, para maximizar el impacto del conjunto de entidades de Economía Social sobre los ODS, en el sentido de que los gobiernos deben implementar políticas públicas amplias y efectivas que fomenten el desarrollo de estas empresas (Chaves Ávila y Gallego Bono, 2020), especialmente teniendo en cuenta las dificultades que se pueden encontrar en épocas de crisis (Chaves Ávila y Savall Morera, 2019).

Esta investigación tiene dos objetivos específicos. El primero, es realizar un análisis descriptivo de una cartera de operaciones de financiación a cooperativas de nueva creación, entendiendo por cooperativas de nueva creación aquellas que cuentan con una antigüedad inferior a tres años ${ }^{6}$, avaladas por una SGR española, y reafianzadas por CERSA (Compañía Española de Reafianzamiento). El periodo analizado va desde el año 2003 hasta el año 2012, e incluye dos etapas diferenciadas por la crisis de 2008. La primera etapa sería de crecimiento y desarrollo (2003-2007) y la segunda etapa sería la de la Gran Recesión (2008-2012). Los 
resultados del análisis descriptivo ponen de manifiesto una disminución de la participación de estas empresas en la solicitud de financiación durante la crisis, en consonancia con la disminución general de actividad de las SGR en la financiación de nuevas empresas en dicho periodo (De la Fuente-Cabrero, Castro-Pardo, SanteroSánchez y Laguna-Sánchez, 2019a).

El segundo objetivo lleva a cabo un análisis comparativo entre el ratio de fallido para el sector cooperativo y el resto de sociedades, incluyendo sociedades limitadas, anónimas y autónomos. Los resultados permiten comparar, por diferentes criterios de clasificación (tamaño de la empresa, sector de actividad y destino de los fondos), el coste del fallido para el grupo de las cooperativas y el resto de las formas societarias, mostrando una situación más favorable para estas entidades de Economía Social de forma generalizada, dado que el coste de falencia de las cooperativas se mantiene por debajo del conjunto de empresas avaladas por las SGR durante todo el periodo analizado.

Las conclusiones obtenidas en este trabajo son, por tanto, no solo relevantes para los gestores de SGR, las entidades financieras, y especialmente para las Administraciones Públicas, sino que también se pueden tener en cuenta para estimar la aportación de fondos de provisiones técnicas destinados a apoyar la financiación de iniciativas emprendedoras sostenibles a través de cooperativas, en definitiva, para diseñar e implementar políticas públicas que sirvan para apoyar el desarrollo de las cooperativas como una alternativa para el desarrollo económico sostenible.

La estructura del artículo es la siguiente. Después de presentar la justificación y objetivos del trabajo en la parte de introducción, se muestra la metodología en el segundo apartado. El siguiente, recoge los principales resultados y, finalmente, las conclusiones y discusión se exponen en el cuarto apartado. Se cierra el artículo con las referencias bibliográficas.

\section{Metodología, fuentes de información y variables a analizar}

La aproximación metodológica utilizada en esta investigación ha sido un análisis descriptivo que ha permitido sintetizar, ordenar y categorizar todas las operaciones de financiación a cooperativas de nueva creación avaladas por SGR en España en el periodo 2003-2012. Este análisis es un estudio específico para la forma societaria cooperativa de las PYMEs españolas, que surge como línea de investigación al realizar un análisis genérico para categorizar la totalidad de operaciones de financiación del sector de SGR con empresas de nueva creación con independencia de su forma societaria, cuyos resultados se recogen en De la Fuente-Cabrero et al. $\left(2019^{\mathrm{a}}\right)$.

La base de datos utilizada para el análisis ha sido facilitada por CERSA a 31 de diciembre de 2018 e incluye 24.837 avales a préstamos, créditos y leasing financiero por un total de 2.395 millones de euros. Del total de operaciones de esta base de datos, 256 avales por un total de 27,36 millones de euros se destinaron a la financiación de proyectos de nuevas cooperativas, lo cual representa el 1\% de la actividad de las SGR, tanto en cuantía como en número de avales. A finales de 2018 estaban pendiente de vencimiento el $8 \%$ del riesgo dado que hay operaciones con un vencimiento superior a 10 años. La información suministrada de cada aval incluye el principal garantizado, la fecha de formalización, el riesgo vivo y el importe fallido del mismo. Se consideran fallidos los riesgos o activos morosos cuya recuperación se considere remota después de su análisis individualizado y se han dado de baja del balance siguiendo lo establecido en la legislación (Ministerio de Economía, 2009).

Se ha seguido la metodología utilizada en De la Fuente-Cabrero et al. (2019a) para definir las variables de interés y agrupar los datos de la cartera de avales a cooperativas. Las variables son las siguientes:

- Avales formalizados: Aunque CERSA incluye algunas categorías de avales que no implican flujos de fondos, como los avales técnicos; en esta investigación se han incluido exclusivamente aquellas operaciones que implican flujos monetarios de financiación, es decir: préstamos, créditos y leasing. Las cuales se han agrupado en función de si el destino de la operación era financiar inversión o circulante.

- Riesgo formalizado: Se consideran las cuantías nominales de las operaciones garantizadas.

- Fallido: Cuantía de los avales que son de dudoso cobro, ya han sido provisionados en su totalidad y están calificado ya en la base de datos como avales fallidos.

Los datos de la cartera global del periodo se han agrupado por año, de forma que se diferencian carteras anuales con información relativa al número e importe de avales que se formalizaron, además de las cantidades impagadas, y clasificadas como fallido para cada cartera anual.

El ratio de fallidos de las carteras de avales se calcula, partiendo de las variables anteriores, como el porcentaje fallido de toda la cartera anual, con independencia del año de impago o del año de dotación de la provisión y/o su calificación como fallido. Esta será la variable central de nuestro análisis, que vendrá identificada como ratio o coste de fallido, o coste de falencia.

La caracterización de las carteras se realiza en función de tres variables de clasificación: 
- Tamaño de empresa: la base de datos incluye información sobre el número de empleados, lo cual nos permite asignar un tamaño a la misma de acuerdo a la definición de la UE de PYME: microempresa (de 0 a 9 empleados), pequeña (10 a 49) y mediana (de 50 a 250 empleados).

- Sector de actividad: la base de datos recoge el código CNAE-2009 de cada empresa, reflejando con ello su actividad económica principal. Así, en función del sector de actividad, se ha realizado la siguiente agrupación: sector primario (agricultura), industria (incluyendo distribución de energía, gas, y otros suministros), construcción, comercio al por mayor y al por menor, transporte, hostelería, información y comunicaciones, actividades profesionales, científicas y técnicas; actividades administrativas y servicios auxiliares, educación, otras actividades sanitarias, actividades artísticas y otros servicios ${ }^{7}$.

- Destino de la operación: la base de datos incluye información sobre cuál es el objeto de la operación, es decir, si se trata de un aval financiero o no, entendiendo por aval financiero aquellos que implican la participación en la operación de una entidad de crédito. En nuestro caso, sólo se han elegido aquellas operaciones que se corresponden con avales financieros, diferenciando dos categorías: préstamos destinados a financiar inversión y créditos destinados a financiar circulante, incluyendo estos últimos tanto tesorería como refinanciación de deuda.

\section{Resultados}

\subsection{Análisis descriptivo de operaciones avaladas a cooperativas}

La cartera de préstamos y créditos avalados se ha clasificado en función de las variables de clasificación definidas en el apartado anterior: tamaño de la cooperativa, sector en el que opera y destino de los recursos obtenidos.

En la Tabla 1 se muestra el importe total formalizado para cada grupo, el número de cooperativas avaladas y el importe medio de la operación en función del tamaño de la cooperativa.

Tabla. 1. Importe formalizado y $\mathrm{n}^{\circ}$ operaciones en función del tamaño de empresa

\begin{tabular}{|l|c|c|c|}
\hline & $\begin{array}{c}\text { Importe } \\
\text { formalizado }\end{array}$ & Número avales & $\begin{array}{c}\text { Importe } \\
\text { medio }\end{array}$ \\
\hline Microempresa (0 a 9) & 17.790 .938 & 208 & 85.533 \\
\hline Pequeña (10 a 49) & 6.685 .511 & 34 & 196.633 \\
\hline Mediana (49 a 250) & 2.883 .400 & 14 & 205.957 \\
\hline TOTAL & $\mathbf{2 7 . 3 5 9 . 8 4 9}$ & 256 & 106.874 \\
\hline
\end{tabular}

Fuente: CERSA y elaboración propia

La mayoría de las operaciones avaladas son en cooperativas de tamaño inferior a 9 trabajadores, microempresas. En concreto son 208 operaciones, que supone el $81 \%$ del total. El importe medio formalizado para este grupo es inferior que en cooperativas pequeñas y medianas, en las cuales solo han sido avaladas con 34 y 14 operaciones respectivamente, con un importe medio próximo a los 200.000 euros, una cuantía media que duplica el importe de las microcooperativas.

Con relación al sector de actividad, podemos observar que, si tenemos en cuenta el número de avales, los sectores que más peso tienen son el primario, con casi un 30\% del total de cooperativas financiadas, seguido del sector industrial y el comercial (Tabla 2). Sin embargo, si tenemos en cuenta el peso de cada sector en función del importe formalizado, el sector industrial es el que está en cabeza con prácticamente un 27\%, seguido del primario, con un $17,6 \%$ y educación, con un 16,3\%. Desde ambas perspectivas, destaca la importancia de la financiación avalada en las cooperativas agrícolas (sector primario) e industriales, siendo en el sector servicios más diversa esta financiación.

En el análisis comparativo entre los datos disponibles para cooperativas y para el resto de sociedades, la clasificación por sectores incluye las siguientes salvedades: transporte está agrupado con información y comunicaciones, y el sector servicios incluye, además de "otros servicios", actividades profesionales, científicas y técnicas, actividades administrativas y servicios auxiliares, educación, otras actividades sanitarias y actividades artísticas. Esto ha sido necesario por utilizar en la comparación la referencia de la investigación de De la Fuente-Cabrero et al., 2019a. 
Tabla. 2. Peso en la cartera por sectores de actividad

\begin{tabular}{|l|c|c|c|c|}
\hline \multirow{2}{*}{} & \multicolumn{2}{|l|}{ Importe formalizado (euros) } & \multicolumn{2}{c|}{$\mathbf{N}^{\mathbf{0}}$ avales } \\
\cline { 2 - 5 } & Importe & \% & $\mathbf{N}^{\mathbf{0}}$ & $\%$ \\
\hline Primario & 4.812 .097 & 17,6 & 76 & 29,7 \\
\hline Industria & 7.318 .691 & 26,7 & 48 & 18,7 \\
\hline Construcción & 1.075 .613 & 3,9 & 14 & 5,5 \\
\hline Comercio mayor y menor & 2.432 .330 & 8,9 & 29 & 11,3 \\
\hline Transporte & 634.458 & 2,3 & 6 & 2,3 \\
\hline Hostelería & 1.695 .676 & 6,2 & 17 & 6,6 \\
\hline Información y comunicaciones & 531.730 & 1,9 & 3 & 1,2 \\
\hline $\begin{array}{l}\text { Act. Profesionales, científicas y } \\
\text { técnicas }\end{array}$ & 3.245 .885 & 11,9 & 27 & 10,6 \\
\hline Act. Administrativas y serv.aux & 219.500 & 0,8 & 5 & 1,9 \\
\hline Educación & 4.469 .458 & 16,3 & 15 & 5,9 \\
\hline O. act.sanitarias & 636.157 & 2,3 & 7 & 2,7 \\
\hline Act. Artísticas & 80.000 & 0,3 & 2 & 0,8 \\
\hline Otros servicios & 208.253 & 0,8 & 7 & 2,7 \\
\hline Total & $\mathbf{2 7 . 3 5 9 . 8 4 9}$ & $\mathbf{1 0 0}$ & $\mathbf{2 5 6}$ & $\mathbf{1 0 0}$ \\
\hline & & & & \\
\hline
\end{tabular}

Fuente: CERSA y elaboración propia

Por último, se ha realizado una clasificación de la cartera analizada en función del destino de los fondos y se ha elaborado una comparativa entre el número de operaciones y el importe formalizado para el periodo, en función de si las operaciones han ido destinadas a inversión o a circulante (Tabla 3).

Tabla. 3. Peso según destino de fondos

\begin{tabular}{|l|c|c|c|c|}
\hline \multirow{2}{*}{} & \multicolumn{3}{|c|}{ PESO SEGÚN DESTINO DE FONDOS, } \\
\cline { 2 - 5 } & \multicolumn{2}{|c|}{ Importe formalizado } & \multicolumn{2}{c|}{$\mathbf{N}^{\mathbf{0}}$ operaciones } \\
\cline { 2 - 5 } & Abs. & $\%$ & Abs. & $\%$ \\
\hline Inversión & 21.923 .774 & 80,1 & 78 & 69,5 \\
\hline Circulante & 5.436 .075 & 19,9 & $\mathbf{2 5 6}$ & $\mathbf{1 0 0}$ \\
\hline TOTAL & $\mathbf{2 7 . 3 5 9 . 8 4 9}$ & $\mathbf{1 0 0}$ & & \\
\hline
\end{tabular}

Fuente: CERSA y elaboración propia 
El 70\% de las operaciones financiadas a las cooperativas durante el periodo analizado se ha destinado a operaciones de inversión y ha supuesto el $80 \%$ del importe formalizado. Solo el 30\% de las operaciones financiadas ha sido para circulante, suponiendo el $20 \%$ de la cuantía formalizada. Estos resultados pueden estar relacionados con el ciclo económico analizado, que principalmente ha sido expansivo, y hasta el año 2009 no comienza a verse en las operaciones de financiación una reducción importante, quedando ligada este periodo a operaciones ligadas a circulante.

\section{2. Análisis comparado de créditos fallidos entre cooperativas y empresas no cooperativas}

La financiación de operaciones de nuevas cooperativas a través de los avales de SGR ha superado los 27 millones de euros en un total de 256 operaciones durante el periodo 2003-2012 (Tabla 4). La utilización de las SGR como soporte de la financiación ha sido desigual en los años considerados, y tiene interés analizar la cartera anual para tratar de caracterizar el comportamiento de las cooperativas relativas a esta financiación. Se observa una tendencia decreciente en el número de avales, a excepción del año 2008 donde hay un pequeño repunte, situándose el mínimo de operaciones formalizadas en año 2012, último año del periodo analizado.

Tabla. 4. Número avales, riesgo formalizado, fallido y ratio fallido de nuevas cooperativas.

\begin{tabular}{|c|c|c|c|c|c|}
\hline Año & $\mathbf{N}^{\mathbf{0}}$ & $\begin{array}{c}\text { Riesgo formalizado } \\
\text { (euros) }\end{array}$ & $\begin{array}{c}\text { Fallido } \\
\text { (euros) }\end{array}$ & $\begin{array}{c}\text { Ratio fallido } \\
(\%)\end{array}$ & $\begin{array}{c}\text { Ratio fallido } \\
\text { global* cartera } \\
(\%)\end{array}$ \\
\hline $\mathbf{2 0 0 3}$ & 48 & 4.711 .977 & 42.863 & 0,9 \\
\hline $\mathbf{2 0 0 4}$ & 40 & 4.878 .557 & 635.656 & 13 \\
\hline $\mathbf{2 0 0 5}$ & 29 & 2.900 .877 & 117.273 & 4 \\
\hline $\mathbf{2 0 0 6}$ & 29 & 3.556 .496 & 303.130 & 8,5 \\
\hline $\mathbf{2 0 0 7}$ & 16 & 2.171 .583 & 168.115 & 7,7 \\
\hline $\mathbf{2 0 0 8}$ & 31 & 3.180 .191 & 0 & 0 \\
\hline $\mathbf{2 0 0 9}$ & 11 & 2.336 .946 & 3.845 & 0,2 \\
\hline $\mathbf{2 0 1 0}$ & 22 & 1.435 .276 & 249.273 & 17,4 \\
\hline $\mathbf{2 0 1 1}$ & 20 & 1.234 .445 & 132.538 & 10,7 \\
\hline $\mathbf{2 0 1 2}$ & 10 & 953.500 & 43.737 & 4,6 \\
\hline TOTAL & $\mathbf{2 5 6}$ & $\mathbf{2 7 . 3 5 9 . 8 4 9}$ & $\mathbf{1 . 6 9 6 . 4 2 9}$ & $\mathbf{6 , 2}$ \\
\hline
\end{tabular}

Fuente: CERSA y elaboración propia

La Tabla 4 recoge la información sobre el riesgo formalizado en cada año y el coste del fallido para cada cartera anual de avales de cooperativas, y se ha añadido los datos del coste de fallido anual para la cartera global de avales formalizados (incluye sociedades anónimas y limitadas, autónomos y otros tipos de empresas). Los mayores ratios de fallidos en las carteras anuales de cooperativas se producen en los años 2004, 2010 y 2011, siendo excepcionalmente bajos los de los años 2008 y 2009, primeros años de la crisis económica, cuyas consecuencias, sin embargo, se reflejan en el coste de la falencia de las carteras de los siguientes años. En concreto, el ratio de los avales del año 2010 alcanzó el 17,4\%, mientras que el ratio de fallido en la cartera global más elevado se produjo en el año 2008, siendo un 15,4\%. Teniendo en cuenta las disparidades observadas en el periodo de crisis, se ha calculado el coste medio del fallido entre los años 2008 y 2012, y los valores son del 7,1\% para las cooperativas y del 10,2\% para el global de la cartera. 
El coste total de fallidos para toda la cartera de avales a cooperativas en el periodo considerado es del 6,2\%, 3 puntos porcentuales inferior al global, lo que indica que las cooperativas han tenido un mejor comportamiento que el resto de las empresas avaladas.

La caracterización del ratio de fallido de las operaciones avaladas a cooperativas por años y tamaño de la empresa se presenta en la Tabla 5. La literatura académica encuentra que existe una relación inversa entre el tamaño de la empresa y el nivel de endeudamiento y el coste de la deuda (Acedo, Ayala y Rodríguez, 2005), por lo que cabría esperar que el riesgo de impago sea menor cuando la empresa tiene un mayor tamaño. En nuestro caso, se debería comprobar que el ratio de fallido es menor en cooperativas de tamaño mediano ( $0 \%$ ), que en pequeñas $(8,8 \%)$ y que en microempresas $(6,2 \%)$. Teniendo en cuenta únicamente los datos globales del coste de falencia, no parece una relación inversa, sino en forma de U. Si se mira el detalle del coste por años, en el caso de las cooperativas de tamaño mediano, se observa que sus operaciones avaladas no han fallado, siendo su coste de fallido nulo durante todos los años del periodo analizado. Pero la comparativa entre pequeñas y micro, no es tan clara. A nivel global del periodo, el coste de falencia es menor en la microcooperativas, y se mantiene así en la mayoría de los años, exceptuando los años 2004, 2007 y 2011.

Tabla. 5. Coste de fallido anual para cooperativas según tamaño de la empresa

\begin{tabular}{|l|c|c|c|c|c|c|c|c|c|c|c|}
\hline & $\mathbf{2 0 0 3}$ & $\mathbf{2 0 0 4}$ & $\mathbf{2 0 0 5}$ & $\mathbf{2 0 0 6}$ & $\mathbf{2 0 0 7}$ & $\mathbf{2 0 0 8}$ & $\mathbf{2 0 0 9}$ & $\mathbf{2 0 1 0}$ & $\mathbf{2 0 1 1}$ & $\mathbf{2 0 1 2}$ & Total \\
\hline Micro & 1,0 & 10,0 & 5,7 & 8,9 & 0 & 0 & 0,4 & 29,8 & 10,6 & 4,7 & 6,2 \\
\hline Pequeña & 0 & 18,7 & 0 & 7,5 & 13,3 & 0 & 0,1 & 0,0 & 11,1 & 0 & 8,8 \\
\hline Mediana & 0 & 0 & 0 & 0 & 0 & 0 & 0 & 0 & 0 & 0 & 0 \\
\hline $\begin{array}{l}\text { Media } \\
\text { ponderada }\end{array}$ & 0,9 & 13 & 4 & 8,5 & 7,7 & 0 & 0,2 & 17,4 & 10,7 & 4,6 & 6,2 \\
\hline
\end{tabular}

Fuente: CERSA y elaboración propia

Si comparamos el ratio de fallido anual de la cartera de operaciones formalizadas para nuevas cooperativas con el ratio de fallido global de la cartera, la financiación de las cooperativas resulta más sostenible. En concreto, para el global de empresas, el ratio de fallido medio en microempresas es del $8 \%$ (frente al 6,2\% en cooperativas), para pequeñas empresas del $11,2 \%$ (del $8,8 \%$ en cooperativas) y para empresas medianas del 9,2\% (frente al 0\%) (De la Fuente-Cabrero et al., 2019a), superior por tanto para todas las categorías al de las cooperativas. Los datos globales de la cartera completa tampoco muestran una relación inversa entre tamaño y coste, sino directa, al menos en el total del periodo.

El análisis del coste del fallido de las cooperativas por sector de actividad se presenta en la Tabla 6. La cartera se ha clasificado, de forma agregada, en los siguientes sectores: primario, industria, construcción, comercio, transporte y comunicaciones, hostelería y otros servicios. 
Tabla. 6. Ratio de fallido para cooperativas según sector de actividad

\begin{tabular}{|l|c|c|c|c|c|c|c|c|c|c|c|}
\hline & $\mathbf{2 0 0 3}$ & $\mathbf{2 0 0 4}$ & $\mathbf{2 0 0 5}$ & $\mathbf{2 0 0 6}$ & $\mathbf{2 0 0 7}$ & $\mathbf{2 0 0 8}$ & $\mathbf{2 0 0 9}$ & $\mathbf{2 0 1 0}$ & $\mathbf{2 0 1 1}$ & $\mathbf{2 0 1 2}$ & Total \\
\hline Primario & 0,0 & 27,7 & 0,0 & 0,0 & 0,0 & 0,0 & 0,0 & 0,0 & 0,0 & 0,0 & 6,5 \\
\hline Industria & 0,0 & 11,9 & 0,0 & 5,3 & 0,0 & 0,0 & 1,1 & 0,0 & 40,0 & 87,5 & 5,3 \\
\hline Construcción & 2,4 & 4,3 & 0,0 & 0,0 & 0,0 & 0,0 & 0,0 & 0,0 & 16,6 & 0,0 & 3,8 \\
\hline Comercio & 8,2 & 2,8 & 41,0 & 0,0 & 25,3 & 0,0 & 0,5 & 0,0 & 0,0 & 0,0 & 12,8 \\
\hline $\begin{array}{l}\text { Transporte } \\
\text { comunicaciones }\end{array}$ & 0,0 & 0,0 & 0,0 & 0,0 & 0,0 & 0,0 & 0,0 & 0,0 & 0,0 & 0,0 & 0,0 \\
\hline Hostelería & 0,0 & 20,6 & 35,7 & 3,7 & 0,0 & 0,0 & 0,0 & 73,8 & 32,1 & 0,0 & 23,5 \\
\hline Otros servicios & 0,0 & 0,0 & 0,0 & 18,2 & 0,0 & 0,0 & 0,0 & 0,0 & 0,0 & 0,0 & 2,6 \\
\hline $\begin{array}{l}\text { Media } \\
\text { ponderada }\end{array}$ & 0,9 & 13 & 4 & 8,5 & 7,7 & 0 & 0,2 & 17,4 & 10,7 & 4,6 & 6,2 \\
\hline
\end{tabular}

Fuente: CERSA y elaboración propia

El ratio de fallido para la totalidad del periodo es muy superior en sectores como la hostelería $(23,5 \%)$ y el comercio $(12,8 \%)$, valores muy superiores a la media $(6,2 \%)$ y al siguiente sector en coste de fallido, el sector primario $(6,5 \%)$. Por el contrario, operaciones avaladas en el sector del transporte y las comunicaciones no han tenido fallidos durante el periodo de análisis.

La información detallada por años destaca el ratio de fallido del sector industrial en los años 2011 y 2012, un $40 \%$ y $87,5 \%$ respectivamente, el del sector comercial en el año 2005 , con un $41 \%$, así como el del sector de la hostelería en el año 2010, con un coste de fallido del 73,8\%. Estos datos concretos tan elevados hay que tomarlos con cautela, porque el número de operaciones en las carteras anuales es pequeño en algunos años, y por tanto, podrían reflejar operaciones específicas, reducidas en número pero con un elevado importe asociado.

El análisis comparativo del coste del fallido por sectores entre la cartera de operaciones avaladas a cooperativas y el global de la cartera muestra, en general para todo el periodo y para todos los sectores, que el coste de falencia es inferior en cooperativas. Únicamente en tres sectores el coste de fallido para cooperativas es superior al del resto de sociedades: en el sector primario, en el que para el resto de sociedades es del 2,9\%, respecto al 6,5\% para cooperativas; en el sector comercio, en el que para el resto de sociedades es del 7,4\% y para cooperativas es del 12,8\%; y en el sector hostelero, en el que para el resto de sociedades es del 10,7\% y para cooperativas es del 23,5\% (De la Fuente-Cabrero et al., 2019a).

En relación con el destino de los fondos, el coste del fallido para la cartera de avales formalizados por cooperativas de reciente creación queda reflejado en la Tabla 7. En ella podemos ver que la tasa de fallido para operaciones de inversión es prácticamente superior en todo el periodo, excepto en los años 2005 y 2012. El coste de falencia para operaciones de circulante es del 3,8\% en el total de periodo, frente al 6,8\% del que se corresponde con operaciones de inversión. En el resto de los tipos societarios, sin embargo, el impago se produce principalmente en operaciones destinadas a financiar operaciones de circulante. En este caso el fallido medio del periodo es del 7,8\% en operaciones de inversión y del 11,2\% en operaciones de circulante (De la Fuente-Cabrero et al., 2019a). 
Tabla. 7. Coste del fallido para cooperativas según destino de fondos

\begin{tabular}{|l|c|c|c|c|c|c|c|c|c|c|c|}
\hline & $\mathbf{2 0 0 3}$ & $\mathbf{2 0 0 4}$ & $\mathbf{2 0 0 5}$ & $\mathbf{2 0 0 6}$ & $\mathbf{2 0 0 7}$ & $\mathbf{2 0 0 8}$ & $\mathbf{2 0 0 9}$ & $\mathbf{2 0 1 0}$ & $\mathbf{2 0 1 1}$ & $\mathbf{2 0 1 2}$ & Total \\
\hline Circulante & 0 & 0 & 37,8 & 7,2 & 0 & 0 & 0,15 & 0,1 & 4,9 & 5,6 & 3,8 \\
\hline Inversión & 1,2 & 13,1 & 0,7 & 8,7 & 8,6 & 0 & 0,2 & 49,2 & 18,4 & 0 & 6,8 \\
\hline $\begin{array}{l}\text { Media } \\
\text { ponderada }\end{array}$ & 0,9 & 13 & 4 & 8,5 & 7,7 & 0 & 0,2 & 17,4 & 10,7 & 4,6 & 6,2 \\
\hline
\end{tabular}

Fuente: CERSA y elaboración propia

\section{Conclusiones}

Las cooperativas pueden ser un agente relevante para la puesta en marcha de nuevos proyectos emprendedores dentro de los objetivos de desarrollo sostenible y del marco de la recuperación económica, y en este contexto, las SGR pueden ser un importante apoyo financiando estas iniciativas empresariales con préstamos garantizados a medio y largo plazo. Ahora bien, es importante estimar adecuadamente el coste del fallido de estas operaciones, para que las SGR pueden disponer de los fondos necesarios para su cobertura.

En este sentido llama la atención que, siendo las cooperativas entes con un gran potencial no solo desde el punto de vista social sino también económico, no existan apenas publicaciones respecto a su financiación, en general, y bancaria, en particular. Como hemos podido ver anteriormente, las investigaciones realizadas respecto a las fuentes de financiación de las cooperativas hablan fundamentalmente de la obtención de fondos a través de recursos propios, tales como aportaciones de socios cooperativistas. Sin embargo, en ninguno de los estudios encontrados se hace referencia a la financiación bancaria de las cooperativas, hecho bastante reseñable teniendo en cuenta que en la financiación de sociedades de nueva creación es la principal y más habitual fuente de recursos. En las investigaciones que versan sobre la financiación de PYMES a través de Sociedades de Garantía Recíproca tampoco se han encontrado referencias específicas a las garantías prestadas a cooperativas.

Entre 2003 y 2012, las SGR españolas avalaron un total de 256 operaciones presentadas por cooperativas, por un importe de aproximadamente 27,4 millones de euros. La tasa de fallido general para todo el periodo es de un $6,2 \%$, cifra 3 puntos porcentuales inferior al global de operaciones avaladas por SGR en España en el periodo 2003-2012. Se observa que la tasa de fallido para cada uno de los años varía considerablemente, teniendo sus máximos en los años 2004, 2010 y 2011, y su mínimo curiosamente en 2008, año en el cual comenzó la crisis económica y, en el que la tasa es cero.

El análisis de las carteras anuales aporta información más detallada en función del tamaño, el sector y el destino del aval. Destaca que las cooperativas de tamaño mediano no han tenido fallidos en sus operaciones a lo largo del periodo, y en algunos años concretos, tanto las micro como las pequeñas han destacado por valores altos del coste de falencia. En el caso de los sectores de actividad, la hostelería y el comercio han tenido un peor comportamiento que el resto, muy por encima de la media. Por último, la ratio de fallido siempre es mayor para operaciones de inversión, excepto en los años 2005 y 2012, en los que la mayor tasa de falencia se obtuvo en operaciones de circulante.

Los resultados del estudio pueden ser útiles para los gestores de las SGR, en orden a minimizar el riesgo de las operaciones concedidas, asignando de una forma eficiente su cobertura. También son de utilidad para las Administraciones Públicas, para la toma de decisiones sobre asignación de fondos de cobertura para estas operaciones o para desarrollar políticas de apoyo a empresas con mayores posibilidades de supervivencia. También para las entidades financieras bancos o cajas de ahorro, pueden resultar interesantes para minimizar el riesgo de las operaciones concedidas o disminuir su tasa de mora. Pero en definitiva los resultados son relevantes para avalar futuras iniciativas emprendedoras.

En el futuro sería interesante analizar la tasa de fallido para una cartera con características semejantes de operaciones formalizadas en los años previos a la crisis que está provocando la pandemia y a los años posteriores a ella, para poder comprobar si se repiten las mismas pautas respecto al comportamiento de pago de las cooperativas. 


\section{Referencias bibliográficas}

Acedo, Ayala y Rodríguez (2005) Rentabilidades, endeudamiento y coste de la deuda de las pymes. Análisis empírico de las empresas por tamaños. Revista Europea de Dirección y Economía de la Empresa, 14, 4, pp. 185-200.

Atienza-Montero y Rodríguez-Pacheco. (2018) Empresas capitalistas versus cooperativas: Análisis comparado de resultados económicos y financieros para España en 2008-2015. CIRIEC-España, Revista de Economía Pública, Social y Cooperativa, $\mathrm{n}^{\circ}$ 93, pp. 115-154. https://doi.org/10.7203/CIRIEC-E.93.10730.

Bartoli, Ferri, Murro y Rotondi. (2013) Bank-firm relations and the role of Mutual Guarantee Institutions at the peak of the crisis. Journal of Financial Stability 9, pp. 90-104. https://doi.org/10.1016/j.jfs.2012.03.003.

Bastida, M.; Vaquero García, A.; Cancelo Márquez, M. y Olveira Blanco, A. (2020) Fostering the Sustainable Development Goals from an Ecosystem Conducive to the SE: The Galician's Case. Sustainability, 12, 500. https://doi.org/10.3390/su12020500.

Belmonte, J. L., Guerrero, A. J. M., \& Cabrera, A. F. (2018) La Evolución Del Movimiento Cooperativo Desde Su Origen Hasta La Actualidad a Través De Su Máximo Exponente: La Sociedad Cooperativa. European Scientific Journal, 14 (29), pp. 53. https://doi.org/10.19044/esj.2018.v14n29p53.

Bertoni, F; Martí Pellón, J y Reverte Maya, C (2019) The impact of government-supported participative loans on the growth of entrepreneurial ventures. Research Policy, Elsevier, vol. 48(1), pp. 371-384. https://doi.org/10.1016/j.respol.2018.09.006.

Bretos I, Díaz-Foncea M, Marcuello C. (2020) International Expansion of Social Enterprises as a Catalyst for Scaling up Social Impact across Borders. Sustainability, 12(8):3262. https://doi.org/10.3390/su12083262.

Bretos, I., Díaz-Foncea, M., Marcuello, C. y Marcuello, C. (2018) Cooperativas, capital social y emprendimiento: Una perspectiva teórica. REVESCO. Revista de Estudios Cooperativos, Segundo Cuatrimestre, $\mathrm{N}^{\circ}$ 128, pp. 76-98. https://doi.org/10.5209/REVE.59775.

Briozzo, A y Cardone Riportella, C (2016) Spanish SMEs' Subsidized and Guaranteed Credit during Economic Crisis: A Regional Perspective. Regional Studies, vol 50, issue 3. https://doi.org/10.1080/00343404.2014.926318.

Briozzo, A; Cardone Riportella, C y Trujillo Ponce (2013) Analyzing the role of mutual guarantee societies on bank capital requirements for small and medium-sized enterprises. Journal of Economic Policy Reform, vol 16, issue 2. https://doi.org/10.1080/17487870.2013.801317.

Calderón Milán, M.J., Calderón Milán, B., Barba Sánchez, V. (2020) Labour Inclusion of People with Disabilities: What Role Do the Social and Solidarity Economy Entities Play?. Sustainability, 12(3):1079. https://doi.org/10.3390/su12031079.

Castro-Núñez, R. B., Bandeira, P. y Santero-Sánchez, R. (2020) Social Economy, Gender Equality at Work and the 2030 Agenda: Theory and Evidence from Spain. Sustainability, 12, 5192. https://doi.org/10.3390/su12125192.

Chaves Avila, R. y Gallego Bono, J.R. (2020) Transformative Policies for the Social and Solidarity Economy: The New Generation of Public Policies Fostering the Social Economy in Order to Achieve Sustainable Development Goals. The European and Spanish Cases. Sustainability, 12, 4059. https://doi.org/10.3390/su12104059.

Chaves Avila, R. y Savall Morera, T. (2019) The Social Economy in a Context of Austerity Policies: The Tension between political discourse and implemented policies in Spain. Voluntas, 30, pp. 487-498. https://doi.org/10.1007/s11266018-00075-3.

CESGAR (2019) IX Informe "La financiación de la pyme en España". Resultados año 2019. SGR-Cesgar, Sociedades de Garantía Recíproca.

CESGAR (2021) El papel de las SGR en la recuperación de la economía Española. SGR-Cesgar, Sociedades de Garantía Recíproca.

Columba, F., Gambacorta, L., \& Mistrulli, P. E. (2010) Mutual Guarantee Institutions and small business finance. Journal of Financial Stability, 6(1), pp. 45-54. https://doi.org/10.2139/ssrn.1601928.

Cowling, M. (2010) The role of loan guarantee schemes in alleviating credit rationing in the UK. Journal of Financial Stability, vol. 6, pp. 36-44. https://doi.org/10.1016/j.jfs.2009.05.007.

De la Fuente-Cabrero, C. (2007) Claves de éxito de las sociedades de garantía recíproca en el período 1994-2005. Madrid: Delta Publicaciones.

De la Fuente-Cabrero, C., Laguna-Sánchez, P. y Villacé-Molinero, T. (2013) SME, bad debt, solvency and access to credit during Spain's current economic crisis. ESIC Market. Economic \& Business Journal, 44(2), pp. 191-207.

De la Fuente-Cabrero, C., Castro-Pardo, M., Sánchez-Santero, R., y Sánchez Laguna, P. (2019a) Análisis del fallido en la financiación del emprendimiento. Una perspectiva temporal desde las Sociedades de Garantía Recíproca. Actas XXXIII Congreso Anual AEDEM, Sevilla. Disponible online: https://www.researchgate.net/publication/335096656_Analisis_del fallido_en la financiacion_del emprendimiento Una_perspectiva_temporal_desde_las_Sociedades_de_Garantia_Reciproca.

De la Fuente-Cabrero, C., Castro-Pardo, M., Sánchez-Santero, R., y Sánchez-Laguna, P. (2019b) The Role of Mutual Guarantee Institutions in the Financial Sustainability of New Family-Owned Small Businesses. Sustainability, Vol. 11 , pp. 1-15.

Díaz Duque, J.A. (2013) Las cooperativas y el desarrollo sostenible. Cooperativismo y Desarrollo: COODES, Vol. 1, №. 1, p.p. 53-69. 
Díaz Foncea, M. y Marcuello Servós, C. (2010) Impacto económico de las cooperativas. La generación del empleo en las sociedades cooperativas y su relación con el PIB. CIRIEC-España, Revista de Economía Pública, Social y Cooperativa, núm. 67, pp. 23-44.

Díaz Foncea, M y Marcuello Servós, C. (2013) Evolución del sector cooperativo en España. 4th International CIRIEC Research Conference on the Social Economy. Bélgica.

Escribano Sotos y Pardo García. (2011) Financiación de las PYMES en un contexto internacional: los instrumentos disponibles. Economía industrial, № 380.

Fernández-Guadaño, J., López-Millán, M. y Sarria-Pedroza, J. (2020) Cooperative Entrepreneurship Model for Sustainable Development, Sustainability, 12, No 13, 5462. https://doi.org/10.3390/su12135462.

Fuentes, J; Puig Blanco, F; Bruque Cámara, S. (2008) Los determinantes de la competitividad en las cooperativas. CIRIEC-España, Revista de Economía Pública, Social y Cooperativa, № 61, pp. 233- 249.

Gadea, E. (2005) La Financiación de las Sociedades Cooperativas: un estudio desde la perspectiva de la Ley de Cooperativas del País Vasco. Boletín De La Asociación Internacional De Derecho Cooperativo, 37, pp. 37-53. https://doi.org/10.18543/baidc-37-2003pp37-53.

Hernández, R. (Dir.) (2014) Informe GEM España 2013. Cise/Fundación Xavier de Salas. Madrid, España. Disponible on line: http://www.gem-spain.com/?q=presentacion-informe-2013.

Honohan, P (2010) Partial credit guarantees: principles and practice. Journal of Financial Stability, Volume 6, Issue 1, pp. 1-9.

López- Cózar y Priede. (2015) Identificación de las principales fuentes de financiación empleadas por la empresa social en la actualidad. Economía Agraria y Recursos Naturales, Vol. 15,1, pp. 41-59. https;//doi.org/10.7201/earn.2015.01.03.

Martínez, L. C. S., y García Ochoa, F. G. (2004) Veinticinco años del sistema de garantías recíprocas español. $R A E$. Revista Asturiana de Economía, No 31, pp. 151-176.

Melián Navarro y Campos Climent. (2010) Emprendedurismo y economía social como mecanismos de inserción sociolaboral en tiempos de crisis. REVESCO. Revista de Estudios Cooperativos, № 100, pp. 43-67.

Moyano Fuentes, J; Puig Blanco, F y Bruque Cámara, S (2008) Los determinantes de la competitividad en las cooperativas. CIRIEC-España, Revista de Economía Pública, Social y Cooperativa, No 61, pp. 233-249.

Mozas Moral, A. y Puente Poyatos, R. (2010) Corporate Social Responsibility and its Parallelism with Cooperative Societies. REVESCO. Revista de Estudios Cooperativos, № 103, pp. 75-100.

Naciones Unidas. (2015) The Millennium Development Goals Report 2015. https://www.undp.org/content/dam/undp/library/MDG/english/UNDP_MDG_Report_2015.pdf.

Ono, A; Uesugi, I; Yasuda, Y (2013) Are lending relationships beneficial or harmful for public credit guarantees? Evidence from Japan's Emergency Credit Guarantee Program. Journal of Financial Stability, vol. 9 (2), pp. $151-167$.

Parker (2002) Do Banks Ration Credit to New Enterprises? And Should Governments Intervene?. Scottish Journal of Political Economy, vol 49, issue 2, pp. 162-195. https://doi.org/10.1111/1467-9485.00227.

Pergelova, A y Angulo-Ruiz, F (2014) The impact of government financial support on the performance of new firms: the role of competitive advantage as an intermediate outcome. Entrepreneurship \& Regional Development, 26 (9-10), 663-705. https://doi.org/10.1080/08985626.2014.980757.

Pombo, P., Molina, H. y Ramírez, J. (2006) The European Framework of Guarantee Systems/Schemes: Main Concepts and Characteristics. Journal of Small Medium Enterprise Development, 1, pp. 127-166.

Pombo, P., Molina, H., Ramírez, J. (2007) The American Framework of Guarantee Systems/Schemes? Main Concepts and Characteristics. Journal Of Small Medium Enterprises Development, (4), pp. 39-68.

Pombo, P., Molina, H. y Ramírez, J. (2008) Conceptual Contributions and Characteristics for Classifying Guarantee Systems/Schemes. Segunda Edición. Málaga. Servicio de Publicaciones, Serie Premios Unicaja. Prize UNICAJA for Economic Research.

Povedano, J. G., Sobrino, J. N. R., González, P. P., y Sánchez, H. M. (2013) ¿Los Sistemas de Garantía se orientan al prestatario o a las entidades de crédito? Estudio de la experiencia en Latinoamérica. Estudios de Economía Aplicada, 31(1), pp. 1-20.

Pozuelo, J., Carmona, P. y Martínez, J. (2012) Las sociedades cooperativas y las empresas capitalistas en la Comunidad Valenciana: análisis comparado de su estructura económica y financiera, CIRIEC-España, Revista de Economía Pública, Social y Cooperativa, № 74, pp. 117-147.

Revista Cooperativas agro-alimentarias (2009) $\quad \mathrm{N}^{\mathrm{o}} 4 . \quad$ Disponible on line: https://www.agroalimentarias.coop/ficheros/doc/02740.pdf.

Sanchez Pachón, L.A. (2016) Instrumentos alternativos de financiación para las cooperativas españolas. REVESCO. Revista de Estudios Cooperativos, Tercer Cuatrimestre, $\mathrm{N}^{\mathrm{o}}$ 122, pp. $285-313$. https://doi.org/10.5209/rev_REVE.2016.v122.52023.

Santero-Sánchez, R., De la Fuente-Cabrero, C. y Laguna-Sánchez, P. (2016) Efectos de la crisis sobre la financiación bancaria del emprendimiento. Un análisis de las microempresas españolas desde el sector de las Sociedades de Garantía Recíproca. European Research on Management and Business Economics, 22(2), pp. 88-93. https://doi.org/10.1016/j.iedee.2015.10.006.

Taghizadeh Hesary,Y (2019) Optimal credit guarantee ratio for small and medium-sized enterprises' financing: Evidence from Asia. Economic Analysis and Policy, Elsevier. https://doi.org/10.1016/j.eap.2018.09.011. 
Wang, Zhang, Liu, Cullinan (2016) Loan guarantees and the cost of debt: evidence from China. Applied Economics, 48:38, pp. 3626-3643. https://doi.org/10.1080/00036846.2016.1142658.

Zubiaurre, M.A., Andicoechea, L. y Saitua, A. (2016) Sociedades cooperativas de trabajo asociado versus sociedades de capital. Análisis comparado de fortaleza financiera y rentabilidad en el País Vasco, CIRIEC-España, Revista de Economía Pública, Social y Cooperativa, № 86, pp.155-194. 